\title{
Transient Cyclic Voltammetry: New Theoretical Challenges to Bring up to Date a Famous Electrochemical Lady
}

\author{
Alexander Oleinick, ${ }^{1}$ Irina Svir, ${ }^{1,2}$ and Christian Amatore ${ }^{3,1, *}$ \\ ${ }^{1}$ PASTEUR, Departement de Chimie, Ecole Normale Superieure, PSL University, Sorbonne University, \\ CNRS, 75005 Paris, France \\ ${ }^{2}$ Design Automation Department, Kharkiv National University of Radioelectronics, Nauky Avenue, 14, \\ Kharkiv 61166, Ukraine \\ ${ }^{3}$ State Key Laboratory of Physical Chemistry of Solid Surfaces, College of Chemistry and Chemical \\ Engineering, Xiamen University, Xiamen, 361005, China \\ * Corresponding author: Christian Amatore<christian.amatore@ens.psl.eu>
}

Summary: Cyclic voltammetry has proven its versatile popularity compared to most other electroanalytical methods for investigating electrochemical mechanisms and kinetics. Many textbooks provide clues for understanding and quantify the main features displayed in a voltammogram but this is unfortunately not often useful when confronted to intricate mechanisms as those presently investigated. This brief opinion article discloses a few theoretical challenges that need to be solved to investigate issues associated with modern applications of cyclic voltammetry.

Cyclic voltammetry has proven its invaluable analytical performance especially since the introduction of microelectrodes [1] followed by that of nanoelectrodes [2] has permitted rapid measurements of concentrations and other electrochemical parameters of importance in still solutions, polymers [3], living materials [4], etc. One of the reasons why cyclic voltammetry supplanted the main other electrochemical methods is that, in addition to classical electrochemical data, it offers a wealth of information about mechanistic pathways initiated by electron transfers to or from an electrode surface [5]. Many textbooks and articles exist to provide useful hints for solving elementary mechanistic situations and extract quantitative data about the thermodynamics and kinetics of the few steps whose nature(s) and magnitude(s) influence the voltammetric data [5-7]. However, most mechanistic problems considered in molecular electrochemistry nowadays involve complex competitive and entangled reactions that occur indifferently in solutions and onto the surface of electrodes, quite often with a series of entwined surface-solution sequences. When confronted to such problems, the classical views result inefficient.

For example, we recently introduced and investigated what is now termed the "LavironAmatore" paradox in the case of the electrocatalyzed reduction of benzyl chloride at silver cathodes [8]. Although the critical steps are essentially heterogeneous, involving adsorbed and chemisorbed intermediates, the voltammetric trace displayed none of the expected corresponding features. Conversely, the voltammetric data seemed fully coherent with diffusion/reaction solution processes as if the silver cathodes were playing a strict outersphere role [9]. For this reason, all previous investigators including the discoverers of the electrocatalytic performance of silver cathodes were mystified. Similarly, it was recently established within a wholly different context that under specific circumstances an interplay

\footnotetext{
\# Dedicated to our esteemed friend and colleague, Prof. Dr. Fritz Scholtz, at the occasion of his $65^{\text {th }}$ birthday.
} 
between surface and solution processes may introduce concentration-dependent selfinhibitory effects whose consequences [10] are not coherent with any predictions based on classical electrochemical laws such as Butler-Volmer even in their Marcus-type version or including Savéant's concerted steps [5].

It is presumable that many of such competitive reactions of intermediates exchanged between an electrode surface and the solution which feeds the overall process have remain unnoticed due to the lack of possible theoretical approaches to apprehend their curious behavior. It is our opinion that only careful investigations based on fast and reliable simulation programs prone to handle complex mechanistic sequences competing not only in solution (e.g., as offered by $\operatorname{DigiSim}^{\odot}[11]$ and DigiElech ${ }^{\odot}[12]$ ) but also finely intertwined with heterogeneous inner-sphere chemical and electron-transfer steps such as those that $\mathrm{KISSA}^{\odot}$ can handle in user-friendly ways [13-15]. Alternatively, one may rely on multi-purpose programs such as Comsol $^{\circ}$-multiphysics programs [16] in order to treat such complex electrochemical sequences but this requires a sufficient know-how [10].

Therefore, it may seem that the theoretical resolution taken since the 60s [5] to disentangle any complex mechanistic electrochemical mechanism featuring electron-transfer activation of molecules are reaching their initial goals after $50-60$ years of intense efforts. However, this is just an impression because whatever the complexity of the sequences that may then be simulated, such approaches rely on statistical laws such as Fick's or Nernst-Einstein's laws. This becomes a serious difficulty when considering nanoelectrodes or electrocatalytic nanoparticles in which stochastic reaction probabilities must be considered $[17,18]$ and no more constant "rate constants". In addition, at nano-electroactive elements, the classical notions of diffusion-migration layers and diffuse layers (and more generally electrical field effects) may become hard to define separately $[19,20]$. Treating these issues will require the introduction of new stochastic approaches that are of a fully different type than those developed for understanding single molecule electrochemistry [21-23], and possibly up to a theoretical multiscale continuum involving molecular dynamics and quantum mechanics in addition to transport [24]. Note however that these theoretical efforts don't need to be specifically developed for the treatment of voltammetric situations but they will need to be adaptable to them. This will certainly be done in future driven by nanosciences.

We wish to conclude with a more prosaic but nonetheless difficult problem. It concerns the alterations brought to voltammetric predictions by ohmic drop and time constant or the postcorrection of their distortions [25]. Commercial software products such as DigiSim $^{\odot}$ or DigiElech ${ }^{\odot}$ propose to treat these alterations through introducing a control of the electrode potential by an ad-hoc pure $R C$ series circuit where $C$ is a pure capacitance representing the double layer capacitance and $R$ is the uncompensated solution resistance. This is making sense in electrochemical textbooks but does not resist the usual practice since nowadays molecular electrochemistry is essentially performed at solid and even nanostructured electrode surfaces. Therefore, except for a few cases, background voltammograms are far from those predicted based on constant double layer capacitances over potential windows used in voltammetric studies as soon as they are defined away from the point of zero-charge. Representing the ohmic and time constant alterations by classical $R C$ circuits is thoroughly misleading. Moreover, incorrect evaluations of ohmic drop and capacitive currents may 
introduce severe errors on the interpretation of transient voltammetric data [26]. This is why we did not incorporate such option in $\mathrm{KISSA}^{\odot}$ [15]. Similarly wrong is the current practice of subtracting a background voltammogram recorded independently from the global one, because the capacitive and Faradic currents cross-talk when the Faradic current develops [25, 27]. It seems therefore that the only viable approach consists in modeling double layers by a constant phase element (CPE) [26, 28], or a combination of CPEs, as currently performed in studies of complex electrochemical impedances [29]. This has provided spectacular results for investigations of simple electron-transfer mechanisms [26] so the next step will certainly consist of the introduction of CPEs in numerical simulation programs capable of handling any complex electrochemical sequence. This is presently investigated in the authors' research team.

\section{Acknowledgments}

This work was supported in parts by CNRS, ENS, Sorbonne University and PSL University (UMR 8640 PASTEUR) as well as by LIA CNRS NanoBioCatEchem. CA also greatly acknowledges Xiamen University for his position of Distinguished Professor.

\section{References}

1 Amatore C (1995) In: Rubinstein I (ed) Electrochemistry at Ultramicroelectrodes in "Physical Electrochemistry: Principles, Methods and Applications" (Monographs in electroanalytical chemistry and electrochemistry) M. Dekker, New York. Chap. 4, 131208

2 See e.g.: Watkins JJ, Chen J, White HS, Abruña HD, Maisonhaute E, Amatore C (2003) Zeptomole voltammetric detection and electron-transfer rate measurements using platinum electrodes of nanometer dimensions. Anal Chem 75:3962-3971

3 See e.g.: Pickup PG, Kutner W, Leidner CR, Murray RW (1984) Redox conduction in single and bilayer films of redox polymer. J Am Chem Soc 106:1991-1998

4 See e.g.: Wilson R, Wightman RM (1985) Systemic and nigral application of amphetamine both cause an increase in extracellular concentration of ascorbate in the caudate nucleus of the rat. Brain Res 339:219-226

5 See, e.g.: Savéant JM, Costentin C (2019) Elements of molecular and biomolecular electrochemistry: an electrochemical approach to electron transfer chemistry, 2 nd edn. Wiley, New York

6 See, e.g.: Bard AJ, Faulkner LR (2000) Electrochemical methods: fundamentals and applications, 2nd edn. John Wiley \& Sons, Inc, New York

7 See, e.g.: Amatore C (2000) In: H. Lund, O. Hammerich (eds) Principles and Methods: Basic Concepts in "Organic Electrochemistry". M. Dekker, New-York. Chap. 1, 1-94

8 Klymenko O, Buriez O, Labbé E, Zhan DP, Rondinini S, Tian ZQ, Amatore C (2014) Uncovering a missing link between molecular electrochemistry and electrocatalysis: mechanism of benzyl chloride reduction at silver cathodes. ChemElectroChem 1:227240

9. For a full analysis of the complexity of such cases even in the simplest situation, see: Klymenko OV, Svir I, Amatore C (2014) Molecular electrochemistry and electrocatalysis: A dynamic view. Molecular Phys 112:1273-1283

10 Chen R, Najarian AM, Kurapati N, Balla RJ, Oleinick A, Svir I, Amatore C, McCreery RL, Amemiya S (2018) Self-Inhibitory electron transfer of the Co(III)/Co(II)-complex redox couple at pristine carbon electrode. Anal Chem 90: 11115-11123 
11 Rudolph M, Reddy DP, Feldberg SW (1994) A simulator for cyclic voltammetric responses. Anal Chem 66: 589A-600A

12 Rudolph M (2003) Digital simulations on unequally spaced grids: Part 2. Using the box method by discretization on a transformed equally spaced grid. J Electroanal Chem 543 : 23-39

13 Amatore C, Klymenko O, Svir I (2010) A new strategy for simulation of electrochemical mechanisms involving acute reaction fronts in solution: Principle. Electrochem Commun 12: $1170-1173$

14 Amatore C, Klymenko O, Svir I (2010) A new strategy for simulation of electrochemical mechanisms involving acute reaction fronts in solution: Application to model mechanisms. Electrochem Commun 12: 1165-1169

15 See also: <www.kissagroup.com>.

16 See e.g.: COMSOL Multiphysics, COMSOL, Inc., < $\underline{w w w . c o m s o l . c o m>. ~}$

17 See, e.g.: Amatore C, Grun F, Maisonhaute E (2003) Electrochemistry within a limited number of molecules: delineating the fringe between stochastic and statistical behavior. Angew Chem Int Edn 42: 4944-4947

18 See, e.g.: Singh P, Lemay SG (2016) Stochastic processes in electrochemistry. Anal Chem 88: $5017-5027$

19 Amatore C, Lefrou C (1990) Is cyclic voltammetry above a few hundred kilovolts per second still cyclic voltammetry? J Electroanal Chem 296: 335-358

20 Norton JD, White HS, Feldberg SW (1990) Effect of the electrical double layer on voltammetry at microelectrodes. J Phys Chem 94: 6772-6780

21 Fan FRF, Bard AJ (1995) Electrochemical detection of single molecules. Science 267: 871874

22 Fan FRF, Kwak J, Bard AJ (1996) Single molecule electrochemistry. J Am Chem Soc 118: 9669-9675

23 For a review of important works in this area see: White HS, McKelvey K (2017) Redox cycling in nanogap electrochemical cells. Curr Opin Electrochem 7: 48-53

24 See, e.g.: Li Y, Janik MJ (2019) Recent progress on first-principles simulations of voltammograms. Curr Opin Electrochem 14: 124-132

25 Nadjo L, Savéant JM, Tessier D (1974) Convolution potential sweep voltammetry. III. Effect of sweep rate cyclic voltammetry. J Electroanal Chem 52: 403-412

26 See, e.g.: Charoen-amornkitt P, Suzuki T, Tsushima S (2017) Ohmic resistance and constant phase element effects on cyclic voltammograms using a combined model of mass transport and equivalent circuits. Electrochim Acta 258: 433-441

27 Andrieux CP, Garreau D, Hapiot P, Pinson J, Savéant JM (1988) Fast sweep cyclic voltammetry at ultra-microelectrodes evaluation of the method for fast electrontransfer kinetic measurements. J Electroanal Chem 243: 321-335

28 Aoki KJ, Chen J (2018) Effects of the dipolar double layer on elemental electrode processes at micro- and macro-interfaces. Faraday Discuss 210: 219-234

29 See e.g.: Hirschorn B, Orazem ME, Tribollet B, Vivier V, Frateur I, Musiani M (2010) Determination of effective capacitance and film thickness from constant phase-element parameters. Electrochim Acta 55: 6218-6227 